national drug shortages to facilitate the medication exchange and sharing between organizations to prevent drug wastages and shortages for better patient care.

\section{IDENTIFICATION OF THE INCIDENCE OF ADVERSE DRUG REACTIONS (ADRS) USING NALOXONE AS A TRIGGER TOOL: A RETROSPECTIVE ANALYSIS}

Nissreen Althaqafi, Asmaa Battah, Eman Youssif. Pharmaceutical Care Services Department, King Abdulaziz Medical City Jeddah

\subsection{6/bmjoq-2019-PSF.38}

Background Adverse drug reactions (ADRs) adversely affect patient outcomes, which may cause patients to lose confidence in the healthcare system. Even with the drastic improvement in healthcare practices, detection of ADRs continues to be an important safety tool to ensure patient treatment outcome and safety. The Institute for Healthcare Improvement (IHI) developed a trigger tool as a method to identify possible adverse events from medicine use in the inpatient setting. In this context, we identify naloxone as a trigger tool to detect unreported adverse effects secondary to the use of medicine.

Methods A retrospective chart review of naloxone prescribed to all admitted patients at KAMC-J over 1 year (2016 to 2017) was done to assess the trigger tool efficacy in the identification of ADRs and to assess the appropriate use of naloxone. We defined the appropriate use of naloxone as documentation of the reason for ordering being present and appropriate. The other objective was to determine the proportion of incidents documented through the safety reporting system (SRS).

Results A total of 100 patients who received naloxone orders were identified, for which all were administered in the inpatient setting. The majority of naloxone orders $(n=62,62 \%)$ were to reverse mental status changes, while six patients $(6 \%)$ required intensive care admission. Only four (4\%) cases out of 100 had a documented ADR report through the SRS. The most commonly prescribed dose of naloxone was $0.4 \mathrm{mg}$ $(56 \%)$ followed by $0.2 \mathrm{mg}(23 \%)$. Only two patients received a higher initial dose of $2 \mathrm{mg}$. The majority were prescribed secondary to morphine (IV) or fentanyl (IV or patch), or hydromorphone (PO in patient with end-stage renal disease), and three patients received naloxone secondary to benzodiazepine administration. Two geriatric patients received naloxone without clear justification and they were not on any opioid drugs. The rest of the patients received various doses $(0.04$ to $1.2 \mathrm{mg})$.

Conclusion Using naloxone as a trigger tool is effective in tracing and tackling ADRs in our institution. We found that naloxone administration was often inappropriate. The most common order for naloxone was a $0.4 \mathrm{mg}$ IV push dose, which caused a reversal of analgesia. Development of guidelines and order sets defining the appropriate use of naloxone will help guide healthcare providers on the appropriate ordering of naloxone based on the clinical situation. Although serious ADR reports are minimal in our data (6\%), it did not eliminate the chance of missing important serious reports due to under-reporting.

\section{9 IMPLEMENTING LARGE-SCALE CHANGE OF QUALITY CULTURE: ABU DHABI STORY}

Asma Alameri, Asma Almannaei, Bashir Aden, Jumana Alabed. Healthcare Quality, Department of Health - Abu Dhabi

\subsection{6/bmjoq-2019-PSF.39}

Background The Department of Health - Abu Dhabi (DoH) is the responsible organization that ensures high-quality, sustainable, and accessible care to a population of about 3 million. Improving the quality of care and enhancing patient experience are the top strategic priorities for the DoH and for Abu Dhabi Vision 2020. However, a quality framework had not been put in place to monitor the performance of healthcare providers and measure the quality levels. Moreover, there were variations in the clinical outcomes among the peer group of providers, which affected the patient experience. In order to improve the quality of care and patient experience, DoH introduced Abu Dhabi Quality Index in 2014, a comprehensive quality monitoring framework that is based on the latest thinking and innovative solutions that allows for continuous quality improvements. It consists of Jawda (quality in Arabic) indicators, patient experience survey, and healthcare professional satisfaction survey.

Methods To implement a wide scale of quality change, the DoH developed and implemented a quality improvement framework to enhance quality culture at system level, improve patient safety at the population level, and reduce quality outcome variation among all healthcare providers. This was achieved through a set of quality indicators, Jawda indicators, that measure clinical outcomes, patient safety, and accessibility. The initiation of what to focus on and the vison was around patients' complaints analysis, workshops with stakeholders, and one-to-one meetings. This was a key driving case for change.

Results The latest data collected from 45 hospitals in Abu Dhabi have shown the following results:

- $20 \%$ reduction in unplanned readmission rate for pneumonia.

- $50 \%$ reduction in cardiopulmonary arrest outside critical care.

- $40 \%$ reduction in rate of falls resulting in any injury.

- $30 \%$ reduction in hospital-acquired or worsening pressure ulcer rate.

- $50 \%$ reduction in emergency primary cesarean section rate.

- $40 \%$ reduction in surgical site infection rate for emergency cesarean section.

Conclusion It has been 4 years since the introduction of Jawda indicators to the health sector in Abu Dhabi, and there have been improvements on the average of quality performance and reduction in quality variation among hospitals. More importantly, quality culture, transparency, and accountability were enhanced. The latest improvements to the program include risk adjustments and clinical subspecialty indicators such as stroke, orthopedics, and bariatric surgeries. In 2019, new quality dimensions will be added to the Abu Dhabi Healthcare Quality Index and hospital rankings will be published. 